\title{
Aportes de la lingüística sistémico-funcional para la enseñanza de la lectura y la escritura en la educación superior
}

Ilene Rojas-García

Universidad del Norte, Colombia

zellene7@gmail.com

\section{Resumen}

El objetivo del artículo es realizar una revisión al objeto y la metodología de la lingüistica sistémico-funcional (SFL), en relación con su pertinencia como herramienta para el desarrollo de los procesos textuales en la educación superior. Para ello, se exploran de manera general los conceptos de género y registro, como nociones fundantes de la teoría sistémica, y se enfatiza en los usos sociales del lenguaje. Posteriormente, se reseñan las posibilidades de trabajo desde la pedagogía de género como campo de investigación permanente para mejorar el desempeño de los estudiantes a partir del conocimiento de la lengua en uso, de modo que sea posible democratizar el saber. Por último, se revisará la propuesta del grupo Prodeac como aplicación directa de la SFL en el contexto latinoamericano, señalando la conexión entre la gramática funcional y los procesos de comprensión y producción de textos académicos. La revisión pone de manifiesto la necesidad de abrir el campo de estudio de la SFL hacia el análisis de la multiplicidad y variedad de textos usados en los círculos académicos como aporte para una pedagogía de la lectura y la escritura en la universidad.

\section{Palabras clave}

Lingüística sistémico-funcional; pedagogía universitaria; enseñanza de la lectura; enseñanza de la escritura; educación superior (Fuente: Tesauro de la Unesco).

Recepción: 10/12/2014 | Envío a pares: 11/02/2015 | Aceptación por pares: 01-04-2016 | Aprobación: 17/04/2016 DOI: 10.5294/edu.2016.19.2.1

Para citar este artículo / To reference this article / Para citar este artigo

Rojas-García, I. (2016). Aportes de la lingüistica sistémico-funcional para la enseñanza de la lectura y la escritura en la educación superior. Educ. Educ., 19(2), 185-204. DOI: 10.5294/edu.2016.19.2.1 


\title{
Contributions of Systemic-Functional Linguistics to Teaching Reading and Writing in Higher Education
}

\begin{abstract}
The objective of this article is to review the purpose and methodology behind systemic-functional linguistics (SFL) in relation to its relevance as a tool for the development of textual processes in higher education. To that end, the concepts of gender and registration are explored in a general way, as founding notions of systems theory, and the social uses of language are emphasized. The possibilities for work are then summarized from the standpoint of gender pedagogy as a field of permanent research. The idea is to improve the student's performance based on knowledge of the language in use, thus making it possible to democratize the knowledge in question. As a final point, the proposal of the Prodeac Group is analyzed, as a direct application of SFL in the Latin American context, noting the connection between functional grammar and the processes of comprehending and producing academic texts. The review highlights the need to open the field of study in SFL to an analysis of the multiplicity and variety of texts used in academic circles, as a contribution to the teaching of reading and writing at the college level.
\end{abstract}

\section{Keywords}

Systemic-functional linguistics, university teaching, teaching reading, teaching writing, higher education (Source: Unesco Thesaurus). 


\section{Contribuições da linguística sistémico-funcional para o ensino da leitura e da escrita no ensino superior}

\section{Resumo}

O objetivo deste artigo é realizar uma revisão do objeto e da metodologia da linguística sistêmico-funcional (SFL, por sua sigla em inglês) a respeito da sua relevância como ferramenta para o desenvolvimento dos processos textuais no ensino superior. Para isso, exploram-se, de maneira geral, os conceitos de gênero e registro como noções fundantes da teoria sistêmica, e enfatiza-se nos usos sociais da linguagem. Em seguida, resenham-se as possibilidades de trabalho da pedagogia de gênero como campo de pesquisa permanente para melhorar o desempenho dos estudantes a partir do conhecimento da língua em uso, de modo que seja possivel democratizar o saber. Por último, revisa-se a proposta do grupo Prodeac como aplicação direta da SFL no contexto latino-americano, sinalizando a conexão entre a gramática funcional e os processos de compreensão e produção de textos acadêmicos. A revisão manifesta a necessidade de abrir espaço para o estudo da SFL enfocada na análise da multiplicidade e da variedade de textos usados nos círculos acadêmicos como contribuição para uma pedagogia da leitura e da escrita na universidade.

\section{Palavras-chave}

Linguística sistêmico-funcional, pedagogia universitária, ensino da leitura, ensino da escrita, ensino superior (Fonte: Tesauro da Unesco). 


\section{Introducción}

En los diferentes niveles educativos, los procesos de enseñanza-aprendizaje se encuentran claramente vinculados con la lectura y la escritura. En la educación superior, se propone a los estudiantes acercarse a una diversidad de textos de carácter científico, que requieren mayores niveles de abstracción y la capacidad para interconectar los saberes, de modo que desarrollen el pensamiento científico, se acerquen a otras formas de entender el mundo y comprendan las corrientes de pensamiento sobre las cuales se basa la ciencia objeto de estudio. Será entonces mediante los textos escritos como los estudiantes universitarios podrán incorporarse a la comunidad académica de la disciplina elegida. En este sentido, el desarrollo de la lectura y la escritura no constituye un saber adquirido en la escuela elemental; sino que trasciende también a la universidad, donde se hace necesario acompañar estos procesos, con el fin de que los estudiantes puedan asumir las nuevas situaciones de aprendizaje direccionadas por otros propósitos sociales y mediadas por un lenguaje más abstracto que el utilizado en la vida cotidiana o en la educación básica (Moyano, 2010). Una de las teorías de base para asumir esta tarea es la lingüística sistémico-funcional (SFL, por sus siglas en inglés), vinculada directamente con las prácticas pedagógicas para la enseñanza de la lectura y la escritura en todos los niveles de la escolaridad.

\section{Fundamentos de la lingüística sistémico-funcional}

La SFL tiene sus orígenes en estudios del lenguaje adelantados por Halliday para la enseñanza del inglés como lengua extranjera. Centra su atención en las prácticas socioculturales que reconocen el contexto y la cultura a la hora de adquirir una lengua (Halliday, 1982). Por su parte, Martin y Rose se sustentan en esta teoría para diseñar estrategias de enseñanza y aprendizaje con el objeto de mejorar el desempeño de los niños indígenas australianos en la lectura y escritura (2005, 2008, 2012; Christie,
1999). Estos niños, capaces de expresarse oralmente en lengua inglesa, tenían dificultades para usar los recursos lingüísticos como medio de aprendizaje de la lectura y la escritura. Se incluyen entonces los planteamientos de Bernstein (1990b) para insistir en el papel del contexto social, como brecha que obstaculiza la relación entre el niño y el acceso al saber, en tanto que la escasez de recursos y oportunidades incide también en los procesos de comprensión y producción de textos, lo que a su vez repercute en bajos niveles de desempeño para todas las áreas. A estas condiciones se suma el hecho de que los profesores orientan la enseñanza según la correspondencia fonográfica, limitando las posibilidades de reflexionar en torno al significado profundo del discurso.

La formación de lectores y escritores en la educación superior presenta una situación semejante: los estudiantes, especializados en el pensamiento concreto y el lenguaje de la cotidianidad, deben enfrentarse al lenguaje de la disciplina que han elegido, usando principalmente las herramientas adquiridas en su formación previa. Los discursos de la ciencia se encuentran más ligados al pensamiento abstracto y divergente, así como a construcciones gramaticales complejas y terminología científica; además, su estudio implica la relación con el contexto social e histórico en el que se desarrollan y vinculan otros conceptos del mundo y de las ciencias. Por tanto, en el marco de un programa de acompañamiento en lectura y escritura para la disciplina, se requiere una teoría lingüística de base que sirva como puente para salvar la brecha entre los textos especializados y los estudiantes universitarios. Tal orientación debe responder a un interés general: comprender y producir con eficacia los textos de las disciplinas y usarlos para responder a las demandas de formación profesional. Pero también debe atender tres intereses particulares: 1) el del docente de lengua, quien solo cuenta con sus conocimientos lingüísticos para comprender los textos especializados; 2) el del estudiante, quien no requiere conocimientos lingüísticos profundos, pero sí debe conocer a ca- 
balidad los conceptos de su área del saber; 3) el del docente de la disciplina, quien domina los conceptos pero desconoce las características del discurso con las cuales generar estrategias que promuevan el aprendizaje del estudiante. Se trata, entonces, de una lingüística aplicada capaz de orientar el trabajo colaborativo entre los tres participantes directos de la lectura y la escritura académica.

Se parte entonces de concebir la SFL como una teoría que posibilita mayores herramientas para una pedagogía de la lectura y la escritura en la universidad, en tanto es posible encontrar en ella, no solo una teoría del lenguaje en contextos reales de uso, sino un metalenguaje que va a permitir relacionar las estructuras gramaticales con el ámbito cultural donde se inserta el texto; de este modo, la conexión entre estos permite comprender a profundidad el sentido, y la información podrá ser aprendida, reflexionada y conectada con otros saberes.

\section{El lenguaje como hecho social}

En primera instancia, la SFL sienta sus bases teóricas y metodológicas en la concepción del lenguaje como un hecho social a partir del cual se desarrolla el pensamiento. Como bien lo definieron los estudios pragmáticos (Escandell, 2006) y sociolingüísticos (Moreno, 1998) del siglo XX que sirvieron de base para la SFL, el lenguaje se encuentra fuertemente ligado a la cultura porque el mundo en el que se vive es lo que se expresa en la lengua; de manera que, para explicarla, es indispensable relacionarla con el contexto social en el que se produce (figura 1). Del mismo modo, el lenguaje configura la visión de mundo del ser humano, puesto que los saberes y las ideas van a estar delimitadas por el lexicón que posee. Esta realidad le permitió a Malinowski (1935, citado por Ghio y Fernández, 2008, p. 34) proponer el concepto de contexto de cultura que trasciende la descripción gráfica o fonética, para observar además el trasfondo cultural que permite interpretar y dar sentido tanto a lo que se hace como a lo que se dice. Posteriormente, Halliday (1982) puntualiza el contexto de cultura como una construcción teórica directamente relacionada con el sistema semántico del lenguaje.

Al trasladar estas concepciones al campo del conocimiento científico de las distintas áreas del saber, es posible establecer un paralelo entre el "hecho social" y las comunidades científicas especializadas. Es entonces cuando aparece un tipo de lenguaje que responde, no solo a los usos específicos del campo del conocimiento, sino además, reflexiona sobre el mundo bajo la mirada de un especialista en la materia. En este sentido, el discurso de la disciplina ha sido estipulado por la comunidad científica a la que pertenece y es, al mismo tiempo, de domino exclusivo de los especialistas en cada campo. Dominar tanto los conceptos como las formas de decir propias de las disciplinas garantizará el ingreso a la comunidad académica de manera equitativa y configurará los esquemas de pensamiento que direccionan la forma de ver el mundo. Así, el pensamiento que configura el lenguaje para actuar en sociedad (y viceversa), se asemeja al pensamiento científico que configura el discurso de la disciplina para actuar en la comunidad científica (figura 2).

Figura 1. El lenguaje y sus relaciones en la vida cotidiana

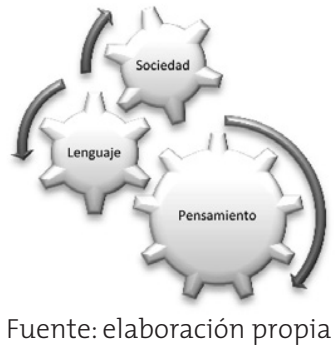

Figura 2. El lenguaje y sus relaciones en la comunidad científica

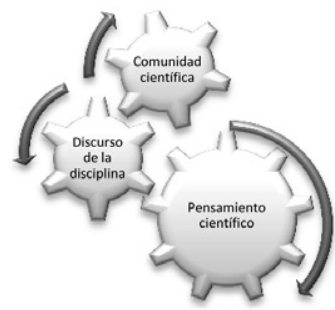

Fuente: elaboración propia 
Para comprender de manera más clara el papel del contexto en el uso del lenguaje, Eggins (1994) propone el ejemplo del forastero que, a pesar de dominar la lengua, requiere ciertos conocimientos culturales y de contexto que le faciliten la comunicación; lo mismo sucede con las bromas en los idiomas extranjeros, para ser comprendidas requieren de tales conocimientos. Situación semejante sucede con los iniciados en un campo del conocimiento quienes, para comprender a cabalidad el discurso, necesitan dominar una serie de saberes previos; esta vez, son más de corte histórico y teórico (si se quiere, enciclopédico), que por lo general debieron ser adquiridos en la educación básica o en cursos de preparación anteriores. La falta de estos saberes resta aportes significativos para la reflexión del tema en su conjunto; también cobra especial importancia la poca familiaridad con la estructura discursiva como causal de incomprensión.

Así pues, la SFL resalta la relación estrecha entre el lenguaje y los usos sociales (científicos) que tienen claramente un impacto directo en el desarrollo del pensamiento. Por tanto, estudiar los textos que se abordan en el aula universitaria desde la perspectiva de la SFL, va a permitir relacionar el contexto científico, sus formas lingüísticas y las visiones de mundo; tres aspectos que deben dominar los estudiantes universitarios para convertirse en profesionales del campo del saber elegido.

\section{El género: concreción del lenguaje como hecho social}

Para comprender las realizaciones lingüísticas y su relación directa con los usos sociales, la SFL plantea el concepto de género para el trabajo en el aula como un

....proceso social, orientado a objetivos y realizado en etapas; social porque inevitablemente estamos tratando de comunicarnos con otros (incluso si ellos no leen o responden inmediatamente a nuestro trabajo), orientado a objetivos porque siempre tenemos un propósito al escribiry nos sentimos frustrados si no lo cumplimos, $y$ realizado en etapas en tanto que usualmente nos toma más de un paso alcanzar nuestros objetivos (Rose y Martin, 2012, p. 54).

Conviene resaltar, además, el género como mecanismo para actuar en el mundo al construir significado y alcanzar un propósito. Esto se evidencia, por ejemplo, con los documentos que se leen y escriben en la universidad, donde el lenguaje media todas las acciones y todos los aprendizajes; se trata de conocimientos mayoritariamente teóricos a los que se accede principalmente desde la lectura de libros científicos o por las explicaciones orales del maestro. De alli que el género es una posibilidad de hacer cosas con el lenguaje, siendo el aprendizaje una actividad psicológica, social, pero especialmente lingüística. En este sentido, Hassan (2011) afirma que:

...nuestro objetivo como maestros es permitir a los estudiantes 'hacer cosas con palabras'. Pero no es posible hacerlo si ellas son producidas como galimatías, usadas de forma que no pueden ser interpretadas. Para hacer cosas con palabras, estas tiene que ser producidas en una relación gramatical que ayude a construir la clase de significado apropiado para la comunidad con el fin de lograr el objeto deseado (p. 15).

Así mismo, Martin (1984, p. 25) describió el género como "una actividad en la que los hablantes se relacionan como miembros de nuestra cultura" (engage as members of our culture), de donde se hace posible enfatizar que solo mediante el dominio de los géneros especializados se accede a la comunidad académica científica de determinado campo.

De este modo, el orden de aparición de los elementos en el texto también es significativo en tanto que, para alcanzar el propósito de la comunicación, mediado eminentemente por el lenguaje, es necesario presentar las ideas a partir de una estructura que ya se encuentra implícitamente convenciona- 
lizada. Se dice "implícitamente" puesto que no se trata de un conocimiento evidente para los hablantes; al igual que la gramática, es una capacidad inconsciente del uso de la lengua que permite emitir y comprender cualquier tipo de enunciados. En palabras de Eggins (1994): "Estamos capacitados para descubrir el propósito general de la interacción porque tenemos - aunque inconscientemente- una idea acerca de cómo se produce una transacción de compra-venta en nuestra cultura, los pasos que tal interacción implica y el tipo de lenguaje que se usa para realizar estos pasos" (p. 6).

Este conocimiento inconsciente se produce incluso en el dominio del lenguaje científico al que se accede conforme se interactúa con los especialistas y con los textos de la comunidad académica; una vez asimilado el código, se usa de manera casi automática. Sin embargo, la experiencia ha demostrado que las competencias para actuar de manera más efectiva con el lenguaje en los distintos contextos académicos, requiere hacer explícitas las estructuras lingüísticas que subyacen a los textos disciplinares, así será posible comprender que "cada etapa y fase del género tiene una función especializada que contribuye al propósito social del género como un todo" (Rose y Martin, 2012, p. 54).

Ghio y Fernández (2008, p. 53) complementan la definición de Rose y Martin al afirmar que "el propósito comunicativo y la función social de los eventos son factores en la caracterización y en la creación de un género". Se enmarca entonces este concepto desde dos realidades: la primera, en relación con la intención del enunciado que determina el sentido y la estructura. La segunda, a nivel de la función social, permite vincularse como miembro activo de una comunidad. En este sentido, el propósito (objetivo) se configura como el elemento fundamental para caracterizar los distintos tipos de géneros que circulan en la vida diaria entre los que se cuentan, por ejemplo, los "académicos, profesionales o de mera interacción social” (Ghio y Fernández, 2008, p. 53).

Teniendo en cuenta las etapas, el propósito y los procesos sociales, los teóricos de la SFL han logrado identificar algunos de los géneros que se utilizan en el aula universitaria (tabla 1).

Tabla 1. Clasificación de géneros

\begin{tabular}{|c|c|c|}
\hline Género & Propósitos & Estadios comunes \\
\hline Reporte descriptivo & $\begin{array}{c}\text { Clasificar y describir elementos del mundo } \\
\text { natural y social }\end{array}$ & Clasificar - describir \\
\hline Explicación & $\begin{array}{l}\text { Explicar procesos del mundo natural y } \\
\text { social }\end{array}$ & Fenómeno-Explicación \\
\hline Recuento histórico & Contar eventos históricos & Registro de eventos pasados \\
\hline Procedimiento & Pasos en experimentos y observaciones & Propósito - Equipo - Pasos \\
\hline Reporte técnico & $\begin{array}{c}\text { Recuento de experimentos y } \\
\text { observaciones }\end{array}$ & $\begin{array}{l}\text { Problemas técnicos - Método - } \\
\text { Resultados - Recomendaciones }\end{array}$ \\
\hline Reporte de investigación & $\begin{array}{c}\text { Describir e interpretar resultados de } \\
\text { actividades de investigación }\end{array}$ & $\begin{array}{c}\text { Problema de investigación - Método - } \\
\text { Resultado - Discusión }\end{array}$ \\
\hline Exposición & Argumentar un punto de vista & $\begin{array}{c}\text { Tesis - Argumentos - Reiteración de } \\
\text { la tesis }\end{array}$ \\
\hline Discusión & Discutir dos o más puntos de vista & Problema - Puntos de vista - Solución \\
\hline Revisión de la literatura & $\begin{array}{c}\text { Discutir múltiples posiciones sobre un } \\
\text { tema }\end{array}$ & Tema - Problema - Solución \\
\hline
\end{tabular}

Fuente: Martin y Rose (2007, p. 3). 
A continuación se presenta un ejemplo de una descripción más detallada sobre el género, retomando el modelo de McCabe (1999).

Un documento legal escrito en el consultorio jurídico de una universidad. El usuario que acude a consultar un caso de violación de sus derechos es interrogado por el abogado de turno a quien le cuenta con detalle los hechos ocurridos. El abogado solicita especificaciones del caso cuando estas no son proporcionadas inicialmente por el usuario, así como las pruebas existentes para respaldar los hechos. El abogado escribe un recurso de reposición para ser leído por el abogado de la contraparte, de modo que le sea posible resolver el caso bajo los parámetros que dictamina la ley.

El relato de los hechos, los actores involucrados, las pruebas y las declaraciones de los afectados orientan la creación de textos que tienen como objetivo informar a otros abogados acerca de la violación de derechos y solicitar los procedimientos pertinentes de conformidad con las leyes. Todo esto con el objetivo de defender o acusar.

De la misma manera, en todas las interacciones sociales ocurren fenómenos comunicativos que incluyen una serie de hechos ya sean orientados por o hacia el lenguaje; es decir, si son actos realizados eminentemente con el lenguaje, como las órdenes o las solicitudes, o si se trata de actividades que terminarán en la configuración de un texto al estilo del ejemplo anterior. Claramente, los procesos de enseñanza-aprendizaje se incluyen dentro de este tipo de interacciones; allí, el lenguaje está presente como actividad (se aprende al escuchar la cátedra del maestro o al leer un documento) y como finalidad (por ejemplo, en la elaboración de un ensayo o en la presentación de un examen). Sin embargo, frente a la variedad de áreas del saber que existen, la lectura y la escritura en el ámbito universitario tienen aún mucho por hacer.

\section{El registro: materialización del género como contexto cultural}

Con el objeto de analizar el género como estrato en el que confluyen el contexto social y la gramática, la SFL diferencia el contexto de cultura del contexto de situación. El primero se refiere al entorno social global, general, que determina los modos de pensamiento y los usos del lenguaje, también entendido como género. Por su parte, el contexto de situación determina el entorno local, particular que rodea al texto, nombrado también con el concepto de registro. En palabras de Halliday (1982), el contexto de situación es una construcción cultural, para explicar cómo un texto se relaciona con los procesos culturales en los cuales se encuentra inmerso. Así, los géneros son realizados a través del lenguaje, pero esta realización se encuentra mediada por el registro (Eggins, 1994).

Como se mencionó en el apartado anterior, el género es un proceso en etapas que se evidencia en la estructura esquemática o la forma como comúnmente se encuentran organizados los textos. Tal organización común, mediada por la intencionalidad, determina el género al que pertenece un texto. Sin embargo, los textos poseen importantes diferencias entre sí que permiten puntualizar su dominio. Aparece entonces el concepto de registro, desde donde se hace posible observar los aspectos fonográficos, lexicogramáticos y semánticos que configuran y materializan el género. El registro "se refiere al hecho de que la lengua que hablamos o escribimos varía de acuerdo con el tipo de situación. [Permite comprender] qué factores de situación determinan qué características lingüísticas" (Halliday, 1982, p. 46). Y a la inversa, a partir de las características lingüísticas se hace posible inferir el contexto de situación que da origen al texto. De allí que Martin y Rose afirmen: "los contextos sociales son realizados como textos, los cuales se realizan como secuencias de cláusulas" (2003, p. 4).

Halliday introduce, entonces, la teoría de registro como las formas de expresión propias de una co- 
munidad, por lo que, si se comprenden los discursos y su forma de estructuración, también será posible comprender el modus operandi de esa comunidad de habla y formar parte de ella. Esto se encuentra en consonancia con los estudios de Bernstein (1990a), quien sostiene que los códigos elaborados permiten "pensar lo impensable", puesto que los significados que ellos presentan, transcienden el espacio, el tiempo y el contexto, y se convierten en significados potenciales de un nuevo orden. En este sentido, al comprender el lenguaje desde su uso específico, se hace posible generar unas estructuras que siguen patrones más o menos homogéneos a partir de los cuales se pueden generar estrategias de comprensión y producción de esos discursos, porque se tiene un conocimiento claro de qué y cómo son.

Figura 3. Materialización del lenguaje como hecho social

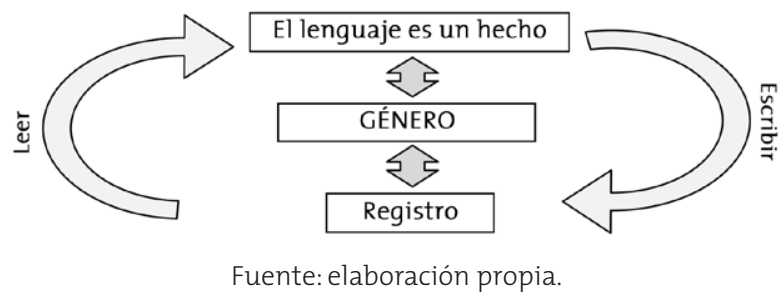

En la figura 3 se muestra el lenguaje como una manifestación social cultural de los grupos humanos que se hace evidente en el género y se materializa en el registro. Al transferirlo al aula, es posible analizar estos aspectos en doble vía: hacia arriba, al momento de enfrentarse a una lectura en la que se parte del material grafológico para inferir de allí los procesos sociales que lo configuran; y hacia abajo, en tanto se parte de un requerimiento social para producir un texto.

Ahora bien, para avanzar en la comprensión del texto, no se requiere observar todos los elementos de la situación en la que ocurre, sino específicamente los aspectos más relevantes, así lo resalta Halliday cuando afirma:
Es importante calificar la noción de "situación" agregándole la palabra "pertinente". El "contexto de situación" no se refiere a todas las porciones de entorno material que podrian aparecer si tuviéramos una grabación sonora y visual de un suceso oral, con todas las imágenes y sonidos que rodean a las expresiones; se refiere a aquellas caracteristicas que son pertinentes al discurso que se está produciendo (1982, p. 42).

Entonces, si el género es un proceso social orientado a fines y llevado a cabo en etapas, el registro va a concretar estos elementos a partir de la realización de tres aspectos más puntuales: el campo, el tenor y el modo. El campo apela a las circunstancias de producción del texto o la situación en donde se produce, incluye además el tema de la conversación. El tenor hace visible la relación entre los participantes y el rol social que interpretan al momento del intercambio comunicativo. Por su parte, el modo permite concretar en el nivel lingüístico las formas gramaticales que configuran el mensaje. Según Eggins (1994), estos componentes no pueden pensarse como instancias separadas sino interdependientes entre sí, las cuales se afectan mutuamente.

Todo lo anterior se corresponde además con las metafunciones del lenguaje definidas por Halliday y Matthiessen (1999) con el ánimo de delimitar los usos del lenguaje en distintas comunidades de habla. La metafunción ideacional se refiere a la representación de la realidad del mundo; la interpersonal asigna los roles sociales que se van a desempeñar al interior del acto de comunicación; la metafunción textual organiza los textos de manera coherente y cohesiva; las tres se manifiestan en la léxico-gramática (tabla 2).

\section{La gramática sistémico-funcional: reflejo del registro como contexto de situación}

El género, el registro y las metafunciones se hacen tangibles en el sistema externo de la lengua. Así, el campo puede observarse a partir del sistema de 
transitividad, el tenor en el sistema de modalidad y el modo en el tema.

El sistema de transitividad se encuentra relacionado con la función ideacional o experiencial, es decir, la función del lenguaje que construye la experiencia de los hablantes sobre su mundo y les permite representar un estado de cosas. En este sistema, la lexicogramática crea una estructura única en la cual, cada constituyente de la cláusula juega un rol específico y significativo (Hassan, 2011). La modalidad, manifestación de la fusión interpersonal, se evidencia en las construcciones gramaticales que definen el intercambio; estas pueden orientarse a dar información, en una cláusula declarativa; solicitar información, a través de la cláusula interrogativa; y ofrecer o demandar bienes y servicios, mediante una cláusula imperativa (Halliday, 2014). Al mismo tiempo, el sistema de valoración permite expresar tonalidades diversas en relación con la actitud y el compromiso frente a lo expresado. Finalmente, el tema, indicador de la metafunción textual, hace referencia a la información que se provee, así como a la distribución de los significados (Ghio y Fernández, 2008).

La importancia de estos distintos componentes al interior de una pedagogía para la lectura y la escritura en el aula universitaria, depende especialmente de las estrategias propuestas por el maestro, en tanto que no se trata únicamente de completar una rejilla donde se identifiquen estos datos, ni de evaluar el dominio de los conceptos en abstracto, sino de establecer procesos de mediación que hagan consciente al estudiante acerca del campo, el tenor y el modo en el uso de la lengua. El análisis de estos componentes va a permitir identificar aspectos ideológicos en el texto que interroguen sobre su adecuación, las relaciones intra e intertextuales, la intención y los componentes gramaticales de la lengua. Por tanto, es claro que no se trata de una gramática prescriptiva que, lejos de juzgar el texto por su corrección, lo incluye dentro de una realidad para analizarlo de manera lógica.
Se trata, entonces, de estudiar los textos especializados de la disciplina como una "gramática que ha evolucionado por siglos en el borde de la ciencia para construir el mundo de manera diferente al que lo hace el habla" (Martin, 1993, p. 134), "está gramática incluye las estrategias para encadenar conceptos, construir argumentos y establecer los límites retóricos y estructurales que se esperan en los géneros científicos y académicos (Ghio y Fernández, 2008, p. 55). Sin embargo, su comprensión se encuentra determinada por la capacidad del lector para reconocer ítems léxicos que determinan el sentido y la intención del texto; un lector novel, poco familiarizado con el contexto de la ciencia, dejará escapar elementos clave para la actuación efectiva a partir del texto escrito.

Por ello, la pertinencia de la SFL como teoría del lenguaje y del aprendizaje, radica en que constituye una herramienta para acceder al saber, de manera que, mientras más acercamiento se tenga con el texto escrito, mayores serán los niveles de conocimiento; $y$, al mismo tiempo, frente a mayores niveles de conocimiento, más accesible será el discurso de la ciencia. En ese sentido, los intereses de la SFL con respecto a los componentes grafofónicos, lexicogramáticos y semánticos de la lengua, permiten comprender los textos de manera más amplia y profunda. Al llevarlos al aula, por ejemplo, los análisis morfológicos de formación de palabras permiten ejercitar a los estudiantes en el dominio del lenguaje especializado; los recursos léxicos le abrirán el campo a los conceptos propios de la disciplina y el nivel gramatical le dará herramientas para comprender y producir las formas de decir de la disciplina; todo lo anterior, al servicio de la construcción de sentido y como respuesta a exigencias de la comunidad académica.

Cabe resaltar, entonces, que no se trata de descripciones meramente gramaticales, bastante estudiadas por las teorías lingüísticas a lo largo de su historia, el interés central se encuentra en observar con detalle el lenguaje para comprender su tejido 
y la intrínseca relación que tiene con el significado Esta posibilidad se logra especialmente a partir de los conceptos que dan nombre a la teoría de la SFL: sistémico-funcional. El primero, da cuenta de los recursos del lenguaje para configurar la experiencia del mundo; el segundo, se refiere al uso a partir del cual los hablantes configuran su experiencia. De alli que la SFL ofrece herramientas para comprender las razones por las cuales un texto posee ciertas características, las opciones alternas que pueden generar otras formas de expresar la misma información y los motivos (individuales o sociales) que el hablante tuvo para generar el enunciado.

\section{La pedagogía de género}

A partir de las aportaciones teóricas de la SFL, Martin y Rose $(2003,2005,2008)$ plantean la pedagogía de género, como mecanismo para mejorar el desempeño de los estudiantes a partir del conocimiento de la lengua en uso, y así, democratizar el saber. En este sentido, Halliday y Martin (1993) fundamentan el lenguaje como la forma prototípica de la semiosis humana, por tanto, el desarrollo del lenguaje es, al mismo tiempo, el desarrollo del aprendizaje: no puede decirse que el lenguaje sea un dominio del conocimiento sino que es la condición esencial para el proceso mediante el cual la experiencia se transforma, deviene en conocimiento. En la misma línea, Hassan (2011) orienta el objetivo de la educación hacia la igualdad de oportunidades para que los estudiantes desarrollen sus habilidades discursivas, precisamente en los aspectos que son esenciales para su educación.

Halliday propone entonces el desarrollo del lenguaje a partir de: 1) el aprendizaje de la lengua, desde los recursos formales (una habilidad que han adquirido todos los nativos de un país); 2) el aprendizaje a través de la lengua, referido al uso del lenguaje como herramienta para articular el sentido de la experiencia y las relaciones sociales con los conocimientos del mundo (una habilidad adquirida por todos los nativos pero que se encuentra determina- da según el dominio del lenguaje); y 3) el aprendizaje sobre el lenguaje, referido al conocimiento consciente de los conceptos fonológicos, gramaticales y de registro, como parte de las actividades del aprendizaje escolar (habilidades adquiridas tras el paso por la educación formal, pero que son poco utilizadas en la vida cotidiana) (Hassan, 2005). Fundamentados en esta concepción, los teóricos de la Escuela de Sidney diseñan los programas Escribir Correctamente y Leer para Aprender a partir de la Teoría de Género (Rose, 2008). Sus aportes se extienden desde la escolarización formal hacia el discurso del trabajo, la industria, la administración, así como a los currículos de geografía, historia y matemáticas (Rose y Martin, 2012). Tales propuestas parten del conocimiento sobre el lenguaje (knowledge about language KAL) que permite comprender las construcciones lingüísticas y hacerlas conscientes dentro del proceso educativo.

En este sentido, el conocimiento y la apropiación del lenguaje en el aula no se produce de manera intuitiva, sino que tendrá en cuenta los planteamientos sobre la teoría de género expuestos en el apartado anterior. No se trata de hacer a los estudiantes expertos en teoría lingüística, sino de ayudarlos a usar los recursos gramaticales para construir generalizaciones y definiciones, para explorar relaciones causales, construir significados hipotéticos acerca de eventos, nombrar y categorizar entidades o eventos (Hassan, 2011). El objetivo es que, tanto docentes como estudiantes adquieran y desarrollen estrategias de lectura y escritura desde las que puedan juzgar la calidad estilística de un texto, su pertinencia para la resolución de casos, las intencionalidades implícitas, así como los usos reales y potenciales.

Como punto de partida general para el análisis de la enseñanza del lenguaje, Rose y Martin (2012) observan las fases sobre las cuales se construye la dinámica de aula. Se inicia con la preparación de los docentes antes de emprender una propuesta pedagógica, es decir, se forman en el dominio del lenguaje (la teoría de género) y su pedagogía (las bases epistemológicas 
ISSN 0123-1294 | Educ.Educ. Vol. 19. No. 2 | Mayo-Agosto de 2016 | pp. 185-204.

Universidad de La Sabana | Facultad de Educación

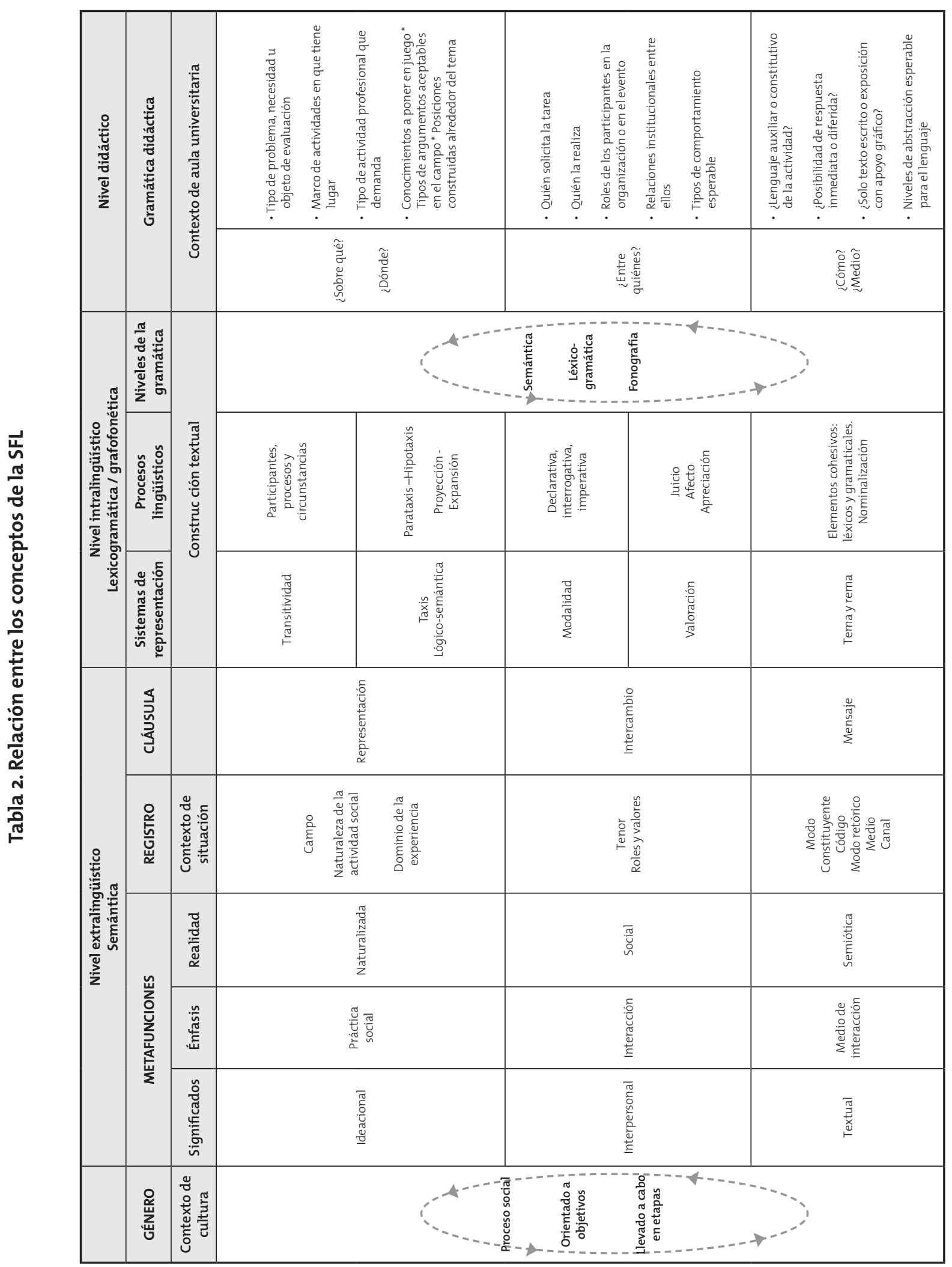

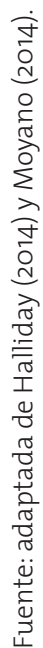


y didácticas requeridas para el trabajo en el aula). Posteriormente, el maestro fija las actividades que se van a desarrollar y planea su discurso, con miras a actuar como mediador entre el estudiante y el conocimiento. En un tercer momento, los estudiantes llevan a cabo la tarea propuesta, concebida como un escaño dentro del proceso de asimilación del saber, que es superado gracias al soporte del docente y al trabajo colaborativo entre compañeros. En este nivel se toman en cuenta el enfoque sociopedagógico de Vygotsky (1998) que resalta el aprendizaje colaborativo, y el currículo en espiral de Bruner (1963), tendiente a retomar y elevar los componentes de cada materia, en una planeación no lineal.

Como aspectos finales en las fases de interacción en el aula aparecen la evaluación y la elaboración. La primera hace referencia a la forma como los docentes valoran las tareas realizadas por los alumnos, que les permite aprender a partir de lo que el constructivismo denomina "error constructivo", es decir, una herramienta de aprendizaje para comprender los procesos de pensamiento que se realizan en la solución de una tarea y se modifican con miras a alcanzar el aprendizaje requerido. La elaboración, por su parte, se trata del siguiente paso en la secuencia de una actividad manual o la comprensión profunda del concepto; permite comprender el saber y motivarse a continuar con el aprendizaje a partir de los logros obtenidos, avalados por el docente. En esta fase adquiere sentido la noción de andamiaje propuesta por Bruner (1983): una metáfora tomada del campo de la industria, para mostrar el rol de los docentes como soporte de sus estudiantes que trabajan en la construcción del dominio de su lengua materna. Inicialmente, el trabajo se desarrolla en conjunto, retomando los postulados de Vigotsky (1978) con respecto a la zona de desarrollo próximo, pero con miras a crear en los estudiantes la autonomía para continuar por sí solos.

Con base en todos estos postulados, la Escuela de Sidney desarrolla el siguiente modelo de enseñanza para los géneros escritos, con el fin de poten- ciar los procesos de aprendizaje en el aula (figura 4). El modelo busca orientar a los estudiantes en la producción de un texto a partir de las habilidades adquiridas en los procesos de lectura. Por ello adicionan actividades que, con la orientación del docente, fluctúan entre la lectura anticipatoria y detallada, hasta la producción de textos. Básicamente, el proceso pedagógico se presenta alrededor de tres fases principales: la deconstrucción, la construcción conjunta y la construcción autónoma. La primera fase busca poner en evidencia el género al que pertenece el texto, con el fin de visualizar sus partes constituyentes como un todo. La segunda fase propone trazar los lineamientos para un nuevo texto teniendo en cuenta los aportes de los estudiantes. La última fase pone la responsabilidad en manos del estudiante para que elabore un texto por su cuenta, a partir de las reflexiones realizadas. La construcción del campo y el establecimiento del contexto son fundamentales en cada etapa del ciclo, los estudiantes las comprenden como una serie de actividades que les permiten aprender acerca del contenido del género y el contexto en el cual se despliega. De esta forma, el objetivo final del ciclo es orientar de manera controlada y crítica la forma como los autores construyen el género (Martin y Rose, 2005).

Figura 4. Modelo de enseñanza de la escritura

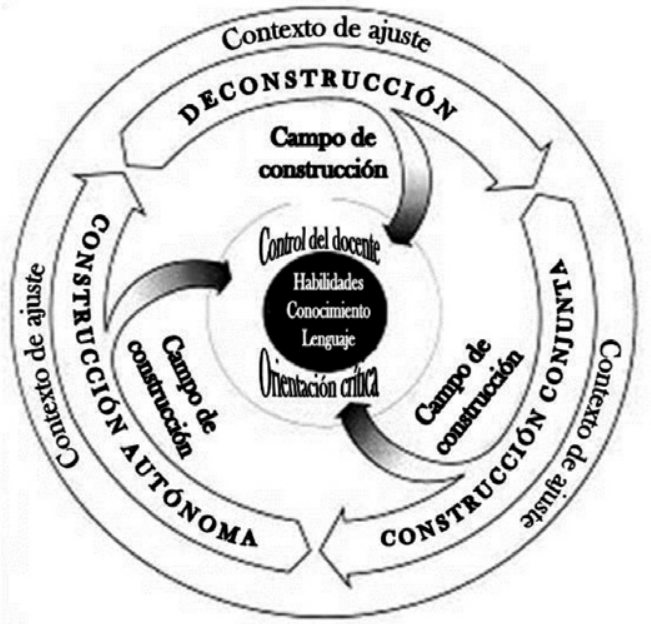

Fuente: Rose y Martin, 2012, p. 308 
Como se evidencia en el esquema, la pedagogía de género resalta los tres componentes en el proceso de enseñanza-aprendizaje de la lectura y la escritura en el aula: el maestro, como mediador entre el saber científico de la lengua y los procesos de análisis que se proponen a los estudiantes para incrementar su dominio del discurso escrito. El estudiante activo, constructor del saber que camina hacia la autonomía, interrogándose sobre la configuración y uso de la lengua. El texto como modelo y objeto de análisis, este es descrito desde la teoría de género en una perspectiva transversal de los procesos de lenguaje como herramienta de acceso a las diferentes áreas del conocimiento. Todo lo anterior confluye dentro de un contexto de interacción y ajuste en el que se desarrolla la tarea y se modifican los aprendizajes.

Es de resaltar el aporte de la pedagogía de género al proponer estrategias de trabajo en el aula y explorar el lenguaje que pueden usar los docentes para hacer explícita su enseñanza; por ello se parte del texto como unidad de significado (nivel semiótico general) que es analizado a partir de sus componentes (nivel léxico-gramatical), de modo que el sentido aparece en relación biunívoca con el sistema de la lengua.

\section{La SLF en la enseñanza-aprendizaje del lenguaje escrito en la universidad}

Tomando como base la teoría de la lingüística sistémico-funcional, y desde el enfoque de la pedagogía de género (Halliday y Martin, 1993; Martin y Rose, 2003, 2005, 2008, 2012), los investigadores de la Escuela de Sidney han adelantado procesos de intervención que sirven de base para la enseñanza y el aprendizaje del lenguaje escrito en el aula universitaria como herramienta para acceder al conocimiento y circular con eficiencia en los discursos de la comunidad académica especializada.

En busca de un enfoque funcionalista que oriente los recursos lingüísticos y discursivos hacia la construcción del conocimiento, así como el análisis de las ideologías que subyacen a las prácticas científicas, el proyecto Prodeac de la Universidad Nacional de General Sarmiento en Argentina encuentra en la SFL el mecanismo para construir, en el aula universitaria, un puente que reduzca la brecha entre la gramática del discurso cotidiano y las construcciones lingüísticas de la ciencia. En este sentido, se retoman los conceptos de género, registro, discurso y lenguaje. Propone, además, el trabajo en equipo entre el docente de lengua, que accederá a los conceptos básicos del saber específico, y los docentes especialistas en cada una de las áreas disciplinares, que se informan sobre las características discursivas de su área; estos orientan a los estudiantes a través de procesos de lectura y escritura programados, evaluados y ejecutados conjuntamente. Los resultados de la implementación del proyecto sugieren que la pedagogía de género facilita al estudiante transferir las herramientas del lenguaje para comprender y producir de manera eficiente los textos de su disciplina, en tanto que responden a exigencias específicas del contexto de uso (Moyano, 2010).

Prodeac retoma también el modelo de enseñanza propuesto por Martin y Rose (2012). Al preparar la intervención didáctica, los investigadores de Prodeac utilizan sus conocimientos lingüísticos para estudiar y describir los textos que serán objeto de discusión en el aula; se enfocan en el reconocimiento de los distintos estratos textuales (campo, tenor y modo), y desde allí orientan la comprensión y producción de textos disciplinares. Además, se ha avanzado en identificar la estructura genérica, la estructura textual y las características "generales" del lenguaje científico en textos de los programas que ofrece la Universidad Nacional de General Sarmiento. Lo anterior en respuesta a la variedad y cantidad de textos usados en la educación superior que exige proponer estrategias de manera muy particular según el tipo de escrito, el objetivo y la tarea. El derrotero para la elaboración de este análisis puede verse en la última columna de la tabla 2 del apartado anterior. 
Sin embargo, a pesar de los múltiples avances en el análisis de textos y las propuestas pedagógicas construidas por la Escuela de Sidney, el grupo Prodeac y las distintas investigaciones que se han suscitado desde diferentes enfoques (Bazerman et al., 2005, 2012; Thaiss et al., 2012), queda mucho por hacer con respecto a la enseñanza del lenguaje en el aula universitaria. Aún hoy, se considera "poco frecuente la enseñanza explícita y curricularizada" de la lectura y la escritura científico-académica, por lo que se requiere un "entrenamiento y formación en didáctica de la lectura y la escritura en las disciplinas para identificar las dimensiones del lenguaje involucradas en las producciones letradas, utilizar un metalenguaje para orientar el trabajo y andamiar y ejercitar el ingreso a las prácticas retóricas más complejas" (Navarro, 2013, pp. 724-725).

Lo anterior obliga a ampliar los estudios sobre los textos que se leen y se escriben, no solo dentro de la formación académica, con el propósito de generar conocimiento, sino al interior del campo laboral, como mecanismo de actuación en los distintos contextos de la cultura. El estudio de esta multiplicidad de géneros textuales tiene varias implicaciones: 1) requiere identificar, en compañía del docente disciplinar, aquellos documentos de mayor relevancia, tanto por su valor teórico para el saber específico, como por sus niveles de uso en los distintos escenarios académicos; 2) describir estos documentos en todos los estratos del lenguaje, con el fin de que el docente de lengua, el docente de la disciplina y el estudiante puedan acceder, desde sus intereses particulares, a las herramientas del lenguaje que harán más efectivos sus procesos de comprensión y producción de textos; 3 ) un trabajo conjunto que integre los conocimientos lingüísticos al campo de la academia y la profesionalización; este trabajo ha de realizarse desde una didáctica que responda a las necesidades de cada comunidad educativa específica, pues, al decir de Gruber (2012), es claro el riesgo de generalizar los resultados de un contexto a otro, ya sea este de la educación básica a la superior, del campo educativo al profesional o de investigaciones en comunidades de lenguas distintas.

Cabe resaltar que tanto el trabajo de descripción y análisis textual como el de su aplicación práctica en el aula involucran "todos los aspectos de la lengua", de allí la importancia de concebir la lectura y la escritura desde el género como un todo. No se trata solamente de ubicar palabras de referencia que permitan determinar si el texto pertenece a uno u otro género; tampoco es suficiente con revisar descripciones formales de textos como demandas, actas o historias clínicas, cuyos modelos se encuentran fácilmente en documentos especializados o en páginas web. "Se debe caracterizar, no solo la estructura del género y la función de sus partes, sino también la organización discursiva de cada parte y algunos aspectos lingüísticos, especialmente cuando se trata de géneros poco estudiados" (Moyano, 2010, p. 472). Una lectura enfocada hacia procesos de significación va a permitir el reconocimiento y la apropiación de las características textuales, garantizando la participación activa en el proceso.

Moyano (2010) llama la atención sobre la necesidad de estudiar los textos a nivel de la valoración y la apreciación. La primera, con el objeto de analizar los efectos retóricos que surgen de las combinaciones de recursos lingüísticos que el autor utiliza para interactuar con el texto y con su lector potencial. La segunda, para reflexionar sobre la construcción del juicio, la gradación y el foco en el discurso de las disciplinas. Es de especial relevancia estudiar los aspectos textuales que más se les dificulta comprender a los estudiantes universitarios y la forma como pueden resolverse desde los avances teóricos de la SFL, como respuesta a las necesidades específicas de formación a este nivel. Quizás el mayor reto para estas exigencias de la enseñanza es que se configuran sobre procesos de pensamiento abstracto y metalingüístico que hacen más complejo, no solo su ejecución como actividades en el aula, sino también las maneras de verificar los alcances obtenidos por los estudiantes, pues se trata de procesos de 
pensamiento que ocurren de manera distinta en cada uno y dependen del tipo de texto, del nivel de análisis y saberes previos de los estudiantes, y de la mediación del docente. Por tanto, es claro que no pueden vincularse como conceptos para ser aprendidos, sino como herramientas para desplegar el análisis que se orienta a la comprensión y producción profunda del texto.

\section{Conclusiones}

Al proponer una reflexión sobre los aportes de la SFL a la alfabetización académica, se evidencia la pertinencia de una perspectiva funcional orientada a fomentar procesos de conciencia y reflexión lingüística, no con el interés de formar teóricos del lenguaje, sino profesionales capaces de actuar de manera eficiente a través de la palabra escrita. Si se asume que el lenguaje forma parte sustancial en la transmisión del saber científico, es claro que los docentes de cualquier área no solo enseñan el saber disciplinar, sino que enseñan también un lenguaje especializado. En este sentido, los avances de la SFL arrojan conocimientos útiles para empezar a comprender en profundidad cómo es ese lenguaje de la disciplina y de qué forma pueden establecerse conexiones didácticas entre el saber científico y las estrategias de lectura y escritura que requieren los estudiantes universitarios. Esto se demuestra en el recorrido mismo de la SFL como proceso dialéctico que transcurre entre: 1) el lenguaje y la sociedad, 2) la lengua como proceso y como sistema, 3) el lenguaje formal y el lenguaje funcional y 4) el lenguaje y el aprendizaje (Hassan, Matthiessen y Webster, 2005).

En consonancia con las corrientes norteamericanas y francesas del análisis del discurso, la perspectiva australiana se instala en un enfoque discursivo y funcionalista del lenguaje que amplía la perspectiva de la acción, postulando el concepto de género a nivel social, procesual e intencional. Pero a diferencia de estas corrientes, la SFL presta especial atención a la gramática como una herramienta concreta al servicio de los usos sociales y del significado.
Así, desde una perspectiva sistémico-funcional, el análisis del discurso transita entre la comprensión del lenguaje en el marco de un contexto cultural y de situación que lo vincula directamente con la realidad, y trasciende hacia la reflexión sobre los recursos del lenguaje totalmente tangibles en el texto que sustentan el análisis.

En clave didáctica, la propuesta metodológica de la pedagogía de género basada en la SFL se preocupa por el reconocimiento, tanto de los fenómenos sociales (relación entre participantes, contexto de situación, intenciones) como de la forma en que estos afectan y son afectados por las construcciones lingüísticas. De tal manera, el estudio del género, el registro y sus componentes constitutivos de campo, tenor y modo, correspondientes a la metafunción ideacional, interpersonal y textual, permiten formarse una idea clara de la manera como se hace posible actuar con las palabras. Para el caso de la educación superior, el análisis del discurso está direccionado al contexto académico, es decir, textos de carácter científico, orientados a explicar hechos y argumentar posturas; usados para aprender y para posicionarse frente a la realidad. A nivel del aprendizaje, el estudio del sistema de Tema-Rema y de Periodicidad correspondiente al campo en la SFL facilita recordar la información pues se hace posible estructurarla en ideas que siguen una secuencia lógica, relacionarla con otros conocimientos y ubicar los datos en el contexto histórico y cultural que los configura; en cuanto a la escritura, estos sistemas permiten reconocer y emular las estructuras convencionalizadas que caracterizan los discursos. Con respecto a la reflexión crítica, el análisis del tenor de la metafunción interpersonal permite reconocer la ideología que subyace a cada texto según sus relaciones de estructuración interna, pero también a partir de sus conexiones con el contexto; en materia de escritura, la conciencia del lenguaje aporta al logro de la adecuación del discurso con el objeto de alcanzar los propósitos comunicativos. Además, los análisis del género y el registro de textos propios de 
la academia contribuyen con el dominio del campo, no solamente desde un saber conceptual, sino de relaciones lógicas entre las temáticas objeto de estudio.

Los aportes de Bernstein, Bruner y Vigotsky a la pedagogía de género proporcionan, además, pautas de trabajo para generar estrategias de interacción en el aula, pues es claro que, si la lengua tiene una función social, su enseñanza se encuentra íntimamente relacionada con la interacción que surja entre el docente y los estudiantes, y con los estudiantes entre sí. De allí que la pedagogía de género, fundamentada en la SFL, se encuentre en la posibilidad de determinar qué, cómo y para qué enseñar lectura y escritura en la educación superior. Los elementos de análisis de una gramática funcional dan pautas a los docentes para comprender cómo lucen los textos cuando son escritos por expertos en libros publicados, cómo se diferencian de los reportes que se solicitan a los estudiantes en una actividad académica, y cuáles son las diferencias con los documentos de los estudiantes con mayor experticia (Hassan, 2011). En tanto que no se trata solamente de enseñar formas de la gramática en abstracto, el enfoque desde la SFL permite a los estudiantes desarrollar una conciencia del lenguaje que trascienda hacia otras esferas de la vida social.

No se trata, entonces, de enseñar únicamente un metalenguaje, tampoco de completar rejillas o subrayar los fragmentos del texto donde aparezcan elementos del campo, tenor y modo, sino de ser conscientes de las posibilidades de uso de la lengua en situaciones de aprendizaje y reflexión. Enseñar a leer y escribir en el contexto universitario tiene que ver con facilitar herramientas para comprender la intencionalidad del autor, la organización del discurso, la interrelación entre las ideas del mismo texto y de este con otros, y utilizar estas herramientas también para la producción escrita.
Sin embargo, existe aún un reto bastante amplio para la SFL y, en general, para los estudios del lenguaje, se trata de trascender hacia la comprensión de los discursos de las disciplinas. No es posible limitarse al análisis del discurso político, escolar, divulgativo o cotidiano; es necesario acompañar los textos puntuales que se trabajan en cada una de las áreas del saber, las enciclopedias médicas del área de la salud, las sentencias judiciales en los programas de derecho, explicaciones matemáticas de las ingenierías, entre otro universo de textos cuyas características genéricas difieren en gran medida de aquellos que están acostumbrados a leer los estudiantes. De esta manera, no solo se estarán facilitando herramientas para el dominio del texto, sino que a medida que lee y escribe, el estudiante aprende el contenido y se familiariza con el discurso. Para lograrlo se requiere de un trabajo transdisciplinar, en palabras de Martin (2000), donde los docentes se conviertan en analistas plurilingües: de las ciencias del lenguaje, las disciplinas específicas y la pedagogía en el aula universitaria. Esta tarea requiere un permanente ir y venir desde la práctica hacia la teoría y de allí de regreso a la práctica y al diálogo con otros investigadores. Cuanto mayor sea el domino de los lingüistas sobre el campo del saber especializado, mayores serán las posibilidades de comprensión de los textos; mientras mayores sean los conocimientos pedagógicos sobre las construcciones lingüísticas, más eficientes serán las propuestas de aula; mientras mejor sea la comprensión de las estructuras textuales de los profesionales en las distintas áreas del saber, mayores serán sus herramientas como mediadores para abrir las puertas a la comunidad académica. Por tanto, se trata de un esfuerzo coordinado entre las distintas áreas que componen las posibilidades de trabajo con el lenguaje como mecanismo para potencializar el aprendizaje y la actuación, que ya ha sido iniciado por la SFL. 
ISSN $0123-1294$ | Educ.Educ. Vol. 19. No. 2 | Mayo-Agosto de 2016 | pp. 185-204.

Universidad de La Sabana | Facultad de Educación

\section{Referencias}

Bazerman, C., Little, J., Bethel, L., Chavkin, T., Fouquette, D.y Garufis, J. (2005). Reference guide to writing across the curriculum. Colorado: Parlor Press.

Bazerman, C., Dean, C., Early, J., Lunsford, K., Null, S., Rogers, P.y Stansell, A. (2012). International advances in writing research. Cultures, places, measures. Colorado: Parlor Press.

Bernstein, B. (1990a). Class, codes and control. New York: Routledge.

Bernstein, B. (1990b). The Structuring of Pedagogic Discourse. London: Routledge.

Bruner, J. (1963a). El proceso de la educación. México: Uteha

Bruner, J. (1983b). Children's Talk: Learning to Use Language. Oxford: Oxford University Press.

Christie, F. (ed.) (1999). Pedagogy and the shaping of consciousness. Linguistic and social processes. London: Continuum.

Eggins, S. (1994). Contexto de cultura: género. Recuperado de http://www.ungs.edu.ar/cienciaydiscurso/wpcontent/uploads/2011/11/Eggins-CAP-2-G\%C3\%Agnero.pdf

Escandell, M. V. (2006). Introducción a la pragmática. Barcelona: Ariel.

Ghio, E.y Fernández, D. (2008). Lingüistica sistémico-funcional. Aplicaciones a la lengua española. Santa Fe: Universidad Nacional del Litoral.

Gruber, H. (2012). The academic writing research group at the University of Vienna. En Thaiss, C., Bräuer, G., Carlino, P., Ganobcsik, L.y Sinha, A. (eds.). Writing programs worldwide. Colorado: Parlor Press.

Halliday, M. A. K. (1982). El lenguaje como semiótica social. México: Fondo de Cultura Económica.

Halliday, M. A. K. (2014). Halliday's introduction to functional grammar. New York: Routledge.

Halliday, M. A. K. y Martin, J. R. (1993). Writing science: Literacy and discursive power. Pittsburgh: University of Pittsburgh Press.

Halliday, M. A. K.y Matthiessen, Ch. (1999). Construing experience through meaning. A language based approach to cognition. London: Continuum International.

Hassan, R. (2011). Language and Education. Learning and teaching in society. Great Britain: Equinox.

Hassan, R., Matthiessen, C.y Webster, J. (2005). Continuing discourse on language. A functional perspective. Great Britain: Equinox. 
Martin, J. R. (1984). Language, register and genre. En Christie, F. (ed.). Children:Writing: Reader (pp. 21-29). Geelong: Deakin University Press.

Martin, J. R. (1993). A contextual theory of language. En Cope, B. y Kalantzis, M. The powers of literacy: A genre approach to teaching writing. London: The Falmer Press.

Martin, J. R. (2000). La gramática se reúne con el género. Reflexiones sobre la Escuela de Sydney. Recuperado de http://www.ungs.edu.ar/cienciaydiscurso/wp-content/uploads/2011/11/La-Gram\%C3\%A1tica-sere\%C3\%BAne-con-el-g\%C3\%Agnero-Jim-Martin1.pdf

Martin, J. R. (2001). Language, register and genre. En Burns, A. y Coffin, C. (eds.). Analysing English in a Global Context: A Reader. London: Routlege.

Martin, J. R. y Rose, D. (2003). Working with discourse. Meaning beyond the clause. London: Continuum.

Martin, J. R. y Rose, D. (2005). Designing literacy pedagogy: Scafolding democracy in the classroom. En Webster, J., Matthiessen, C.y Hassan, R. (eds.). Continuing Discourse on Language. London: Equinox.

Martin, J. R.y Rose, D. (2007). Interacting with Text: the role of dialogue in learning to read and write. Recuperado de https://tvo.wikispaces.com/file/view/Interacting+with+Text.pdf.

Martin, J. R. y Rose, D. (2008). Genre relations, mapping cultures. Great Britain: Equinox.

Martin, J. R. y Rose, D. (2012). Learning to Write, Reading to Learn. Australia: Equinox.

McCabe, A. (1999). Theme and thematic patterns in Spanish and English history texts. Recuperado de http:// www.isfla.org/Systemics/Print/Theses/McCabephd.pdf

Moreno Fernández, F. (1998). Principios de sociolingüistica y sociología del lenguaje. Barcelona: Ariel.

Moyano, E. (2010). Escritura académica a lo largo de la carrera: un programa institucional. Revista Signos, 43 (74), 465-488. Recuperado de http://www.scielo.cl/scielo.php?script=sci_issuetoc\&pid=0718093420100005\&lng=es\&nrm=iso

Moyano, E. (2014). Propuesta de desarrollo de habilidades de lectura y escritura en la universidad. Presentación en Taller de Lectura y Escritura en la educación superior. Universidad del Norte.

Navarro, F. (2013). Trayectorias de formación en lectura y escritura disciplinar en carreras universitarias de humanidades. Revista Mexicana de Investigación Educativa, 18 (58), 709-734.

Rose, D. (2008). Reading to learn. Australia: Equinox.

Thaiss, C., Bräuer, G., Carlino, P., Ganobcsik, L.y Sinha, A. (2012). Writing Programs Worldwide. Colorado: Parlor Press. 
ISSN 0123-1294 | Educ.Educ. Vol. 19. No. 2 | Mayo-Agosto de 2016 | pp. 185-204.

Universidad de La Sabana | Facultad de Educación

Trosborg, A. (2002). Discourse analysis as part of translator training. En Schäffner, C. (ed.). The Role of Discourse Analysis for Translation and Translator Training. Great Britain: MultilingualMatters.

Vygotsky, L. S. (1998). Pensamiento y lenguaje. La Habana: Editorial Pueblo y Educación.

Vygotsky, L. S. (1978). Mind in Society: the Development of Higher Psychological Processes. Cambridge: Harvard University Press. 